\title{
"Tumor immunology meets oncology IV", 23rd and 24th May 2008 Martin Luther University Halle-Wittenberg, Halle, Germany
}

\author{
Juergen Bukur · Dennis Loeffler $\cdot$ Chiara Massa $\cdot$ \\ Lutz Mueller · Dagmar Riemann • \\ Hans-Joachim Schmoll · Barbara Seliger
}

Received: 30 June 2008 / Accepted: 14 August 2008 / Published online: 28 August 2008

(C) Springer-Verlag 2008

In May 2008 the fourth conference of "Tumor immunology meets oncology" was held at the University Hospital of the Martin Luther University Halle-Wittenberg in Halle organized by the Institute of Medical Immunology (director Barbara Seliger) together with Hans-Joachim Schmoll the director of the Department of Internal Medicine IV. The program of this conference focused on the mechanisms used by tumor cells to escape from immune effectors and/or suppress immune responses and on different immunotherapeutic approaches developed to circumvent immune escape ranging from the identification of novel target structures to the usage of mesenchymal stem cells (MSC) as a novel therapeutic modality.

Tumor cells can evade recognition from immune cells by various strategies like secretion of immune suppressive cytokines and mediators, expression of FAS ligand, lack of costimulatory molecules as well as abnormalities of MHC class I surface expression, which could lead to an impaired immune response, suppression of immune effector cells, defective tumor cell recognition and finally to tumor cell resistance to cell death. The first talk by Barbara Seliger focused on the molecular mechanisms of MHC class I deficiencies in different tumor histotypes. Both patients' biopsies and tumor-derived cell lines presented a high

J. Bukur $\cdot$ D. Loeffler $\cdot$ C. Massa $\cdot$ D. Riemann $\cdot$ B. Seliger $(\bowtie)$ Institute of Medical Immunology,

Martin-Luther-University Halle Wittenberg,

Magdeburger Str. 2, 06112 Halle (Saale), Germany

e-mail: barbara.seliger@medizin.uni-halle.de

L. Mueller $\cdot$ H.-J. Schmoll

Department of Internal Medicine IV,

Oncology/Hematology,

Martin-Luther-University Halle-Wittenberg,

Halle (Saale), Germany frequency of downregulation or loss of MHC class I surface expression that correlates with a diminished $\mathrm{T}$ cell response, disease progression as well as reduced patients' survival [2]. These MHC class I abnormalities were mainly associated with a dysregulated expression of one or more components of the MHC class I antigen processing machinery (APM) rather than with structural alterations. B. Seliger demonstrated that these dysregulations could occur at the epigenetic, transcriptional and/or posttranscriptional level mainly affecting the transporter associated with antigen processing (TAP) subunits, tapasin, the MHC class I heavy chain and $\beta_{2}$-microglobulin $\left(\beta_{2}-\mathrm{m}\right)$ as well as interferon (IFN)-responsive genes/proteins. So far, there exists no information concerning a hierarchy of such molecular events, but based on the current knowledge they appear to be tissue/tumor specific.

Recently, research has also focused on the role of some cytosolic as well as the endoplasmic reticulum (ER)-resident aminopeptidases (ERAP)1 and ERAP2 in shaping MHC class I surface antigen expression and consequently the immune response due to their altered expression pattern in tumor cells. Jens Wulfänger (Institute for Medical Immunology, Martin-Luther-University, Halle-Wittenberg, Germany) expanded the repertoire of enzymes involved in regulation of HLA expression levels to the membrane-associated aminopeptidase N (APN/CD13), an enzyme with zinc-dependent ectopeptidase activity, which has been shown to influence the MHC class II antigen presentation [5]. Using wild type (wt)-APN and enzymatically defective mutant (mu)-APN transfectants APN activity decreased cell growth and migration of HEK293 cells in vitro, which was associated with a diminished CXCR4 transcription and protein expression [25].

Based on a recent report in HeLa cells suggesting that activation of CXCR4 reduces HLA class I cell surface 
expression [26] due to an increased ubiquitination of these molecules, one might speculate that wt-APN-mediated CXCR4 suppression should contrast this phenomenon. Indeed, wt-APN overexpression into MHC/APN-negative/ low HT29 colorectal cancer cells caused induction of HLA class I surface antigen expression, which was accompanied by an increased protein expression of various MHC class I APM components, e.g. HLA class I heavy chain, TAP1, TAP2, $\beta_{2}-\mathrm{m}$, the low molecular weight proteins (LMP)2 and LMP7. Immunoprecipitation experiments demonstrated a physical association between APN and HLA class I molecules. Thus, APN can be involved in abrogation of cell proliferation/migration and induction and/or stabilization of HLA class I surface expression.

The importance of HLA antigens for tumor development was underlined by the talk of Giuseppe Masucci (Karolinska University Hospital, Stockholm, Sweden), who correlated the mortality rate of ovarian [6] and prostate cancer as well as melanoma patients with a high frequency of HLA-A2 allele expression in the Swedish population $(>50 \%)$. By statistical analysis the common ancestral haplotype $62.1(\mathrm{~A} 2, \mathrm{~B} 15, \mathrm{Cw} 3, \mathrm{DRB} 1 * 04)$ was identified in the Swedish population as unfavourable for ovarian and melanoma patients leading to an accelerated progression of disease and/or reduced patients' survival. These data suggested that this haplotype is associated with high immune response and is able to control a minimal residual disease by increasing the risk for selection of malignant clones. In addition G. Masucci and co-workers extended the work of B. Seliger and S. Ferrone on MHC class I APM abnormalities to ovarian carcinoma. Immunohistochemical analysis demonstrated a frequent loss or downregulation in MHC class I APM component expression in particular in ovarian carcinoma patients with the haplotype 62.1 , which might at least partially be caused by chromosomal alterations or loss of heterozygosity [16]. Moreover, tumors with HLA class I abnormalities exhibit a reduced lymphocyte infiltration and a lack of p21/waf-1 expression.

Beside the relevance of HLA class I abnormalities for immune escape, the tumor can also suppress existing or developing immune responses either by inducing suppressive cells, like regulatory $\mathrm{T}$ cells $\left(\mathrm{T}_{\text {reg }}\right)$ and myeloid-derived suppressor cells (MDSC), or by the development of an immune suppressive microenviroment. Rolf Kiessling (Cancer Centre, Karolinska Intstitute, Stockholm, Sweden) presented data correlating immune suppression with tumorinduced oxidative stress. In cancer patients indicators for oxidative stress include increased lipid peroxidation, increased DNA damage in lymphocytes, decreased levels of antioxidants in blood plasma and high numbers of cells producing reactive oxygen and nitrogen species (ROS and RNS) either at the tumor site or in peripheral blood. Inducible NO synthase (iNOS) and COX-2 expression levels were elevated in stage III melanoma patients with a shortterm survival when compared to patients with long-term survival suggesting that iNOS and COX-2 represent adverse prognostic factors (C. Johansson, in preparation). This concept is supported by a distinct $\mathrm{H}_{2} \mathrm{O}_{2}$ sensitivity of different effector cell subsets. Among $\mathrm{CD} 8^{+}$antigen-specific $\mathrm{T}$ cells the effector memory subpopulation was the most sensitive to $\mathrm{H}_{2} \mathrm{O}_{2}$-induced cell death $[10,22]$. Similarly, concerning natural killer (NK) cells, the cytotoxic CD56dim NK cells are more sensitive to $\mathrm{H}_{2} \mathrm{O}_{2}$ than CD56bright NK cells, whose lysates have a higher anti-oxidant capacity. Based on these results, new immunotherapeutic approaches were developed employing anti-oxidants to antagonise the oxidative stress in cancer patients. These include vitamin E, which enhances Th-1 responses and NK cell activity in patients with colorectal carcinoma $[1,7,11]$ and histamine dihydrochloride in combination with IL-2 synergistically, which improves the leukemia-free survival in patients with acute myeloid leukaemia (AML) [4]. Based on these studies antigen-specific cytotoxic $\mathrm{T}$ lymphocytes (CTL) were engineered with the recombinant human catalase gene to enhance their tolerance against oxidative stress. CMV-specific catalase expressing T cells increased the survival and retention of IFN- $\gamma$ secretion upon in vitro antigenspecific stimulation in the presence of $\mathrm{H}_{2} \mathrm{O}_{2}$. Thus, catalase expressing $\mathrm{T}$ cells might have the capability to overcome oxidative stress in cancer patients thereby improving the outcome of immunotherapy.

In addition to the tumor-induced immune suppression Vincenzo Bronte (University of Padua, Italy) summarised in the key note lecture the latest data regarding the development, phenotype and activity of MDSC. This previously very heterogeneous cell population derives from a common myeloid progenitor that is expanded during tumor development due to an altered myelopoiesis mediated by tumorderived soluble factors, like GM-CSF. Phenotypically, MDSC were initially characterised by the expression of the markers $\mathrm{Gr} 1$ and $\mathrm{CD} 11 \mathrm{~b}$, but purification through these molecules from mice bearing different tumor types results in very different populations based both on expression of other markers and on degree of immunosuppressive activity. By dissecting the $\mathrm{Gr}^{+}$population, it was found that the suppressive population segregate with the Gr1 intermediate expression. Moreover, experiments with knock out mice indicated a correlation of the suppressing activity with the expression of CD124, the IL- $4 \mathrm{R}_{\mathrm{a}}$ common chain for IL-4 and IL-13 providing responsiveness to IL-13. Indeed, MDSC require for their activation the presence of IFN- $\gamma$ and IL-13, which are then produced in an autocrine loop. Upon stimulation with these cytokines, two enzymes involved in the L-arginine metabolism, namely arginase 1 ( $\arg 1)$ and/or iNOS2 are induced, which is accompanied by a depletion of the amino acid and production of catabolites 
thereby resulting in an impaired $\mathrm{T}$ cell proliferation. The relevant role of this escape mechanism for tumor development and immunotherapy failure derive from both in vitro and in vivo studies, in which the $\mathrm{Gr} 1^{+} \mathrm{CD} 11 \mathrm{~b}^{+}$population is physically depleted or inactivated for example by usage of phosphodiesterase-5 inhibitors. Upon such in vivo treatments it is possible to induce an immune response.

Ena Wang (Infectious Disease and Immunogenetics Section, NIH, Bethesda, USA) molecularly analysed an unique patient model demonstrating that metastatic melanoma is a clonal disease with stem cell persistence [23]. The molecular make up of cell lines generated from metastases of the same melanoma patient occurring at different time points during melanoma recurrence was investigated using microarray-based comparative genomic hybridisation (CGH), mutational analysis of the $\beta$-catenin gene and the human androgen receptor inactivation assay for clonality. These assays highlighted the presence of a consistent core of common genetic traits together with divergent phenotypes suggesting that all metastases were derived from the same primary founder cell clone. However the non-cumulative differences indicate that each cell line was not derived from the most recent previous one in a sequential manner, but followed individual evolutionary pathways throughout the patient's history. These data support the hypothesis of the existence of a common progenitor tumor stem cell giving rise to a genetically unstable progeny that maintains a conserved core of genetic imbalances while adding new, independent ones. More detailed transcriptional and CGH analyses demonstrated a divergent pigmentation pattern, progressive loss of melanoma differentiation antigens, copy number changes and loss of chromosome $9 p$ as well as HLA class I abnormalities and loss of a series of genes associated with IFN function that might have allowed tumor escape from immune recognition in the later stages. Genetic, transcriptional, post-transcriptional and epigenetic patterns individually influenced the development of this patient's tumor and the individuality was maintained through the end stages of disease. In this context it is noteworthy that genomic imbalances detectable by CGH only contribute in $25 \%$ to the transcriptional modulation determining the autologous tumor singularity.

It is well accepted that $\mathrm{T}$ cell-based immunotherapies are less efficient than initially expected. These disappointing results are likely to be caused, at least in part, by the evasion of tumor cells from immune recognition. In order to improve the efficacy of immunotherapies, a general strategy to counteract the diverse immune escape mechanisms utilized by tumor cells has to be developed. The human high molecular weight-melanoma associated antigen (HMWMAA) was chosen by Soldano Ferrone (Cancer Institute, University of Pittsburgh, USA) (1) since it is recognized by antibodies and CTL to mount both humoral and cellular immune responses and (2) since it is expressed by cancer stem cells known to be required for tumor growth. HMWMAA is expressed at a high density on the cell surface of melanoma (85\%), glioma (70\%), chondrosarcoma (50\%) cells and on acute lymphoblastic leukemia (55\%) cells, but has a restricted distribution in normal tissues. This antigen is highly conserved through phylogenetic evolution. Using the mouse anti-idiotypic mAb MK2-23 for the treatment of melanoma patients, all immunized patients developed a high titer of anti-mouse Ig antibodies and $60 \%$ of them produced HMW-MAA-specific antibodies with low titers and affinity [24]. Interestingly, the patients who developed a HMW-MAA-specific humoral immune response had an improved survival rate.

In a variety of mouse models engrafted with different human tumors S. Ferrone and colleagues employed different HMW-MAA-specific vaccination strategies including the HMW-MAA-specific mAb 763.74, HMW-MAA-transfected dendritic cells (DC) and HMW-MAA-recombinant Listeria monocytogenes in order to generate potent humoral as well as cellular-mediated cytotoxic immune responses, which led to tumor regression and increased survival rates of tumor-engrafted mice [9, 23]. Bases on recent data, S. Ferrone stressed the idea that HMW-MAA might be involved in promoting the migration of melanoma cells, which could be blocked by a mixed panel of HMW-MAAspecific $\mathrm{mAb}$. Moreover, flow cytometric analyses of different breast carcinoma cell lines using a combination of HMW-MAA-specific mAbs together with stem cell-specific marker molecules like CD44, CD24 and aldehyde dehydrogenase indicates that HMW-MAA is expressed on breast carcinoma stem cells. These findings are compatible with the possibility that HMW-MAA may be useful to target breast cancer stem cells with humoral and cellular HMWMAA-specific immunotherapy.

To further evaluate how cancer stem cells can evade immune responses, Olivia Doering (Institute of Medical Immunology, Martin-Luther-University, Halle-Wittenberg, Germany) analysed the expression of immune-related molecules on embryonic cancer cells (ECC). During differentiation ECC change the expression of regulatory surface proteins, like B7-H1, paralleling the development of cisplatin resistance and loss of expression of embryonic transcription factors [12]. These results lead to the hypothesis that tumor stem cells bear a specific immunophenotype, which also could represent targets for new therapeutic approaches [3].

Shifting from tumor stem cells to the adult non-malignant mesenchymal stem cells (MSC) Lutz P. Mueller (Department of Medicine IV, Martin Luther University, Halle-Wittemberg, Germany) presented an overview on their possible usage as carriers for anti-tumor activity. The basis for this approach is the observation that non-malignant 
bone-marrow-derived cells like MSC can actively integrate into tumors in vivo $[14,21]$ and thus upon genetic modification can be used for a local therapy. Indeed, transgenic expression of apoptosis-inducing ligands allows MSC to suppress tumor growth in vitro and in vivo [1]. As MSC show a relative resistance to chemotherapy [13] the use of autologous MSC seems feasible for clinical trials. However, depending on the model, the extent of tumor integration of systemically applied transgenic MSC is low and appears to represent the main obstacle for a future clinical use. Methods to enhance directed migration of MSC have recently been described [20].

While the existence of multipotent stem cells like MSC has been demonstrated in the adult organism, recent data support the hypothesis that also pluripotent stem cells exist in the adult organism. The group of Machalinski and coworkers were able to identify these so-called very small embryonic-like (VSEL) stem cells in various tissues including cord blood and peripheral blood $[8,18]$. VSEL stem cells not only show a pluripotent differentiation potential, but also a typical embryonic phenotype characterized by the differentiation-dependent expression of specific surface markers and transcription factors, like Oct-3/4, Nanog, Rex-1 and Rif1 [19]. Based on these features VSEL stem cells may represent a target for malignant transformation in the adult organism.

Permanent cure of AML by chemotherapy alone remains elusive for most patients because of the inability to effectively eradicate leukemic stem cells (LSCs), the self-renewing component of the leukemia. To develop therapies that effectively target LSC, one potential strategy is to identify cell surface markers that can distinguish LSC from normal hematopoietic stem cells. CD96, also known as Tactile first described on activated $\mathrm{T}$ cells represents a promising LSC-specific candidate antigen. Martin Gramatzki (Christian-Albrecht University of Kiel, Division of Stem Cell Transplantation and Immunotherapy, Kiel, Germany) reported that $\mathrm{CD} 96$ is expressed in many cases on the majority of $\mathrm{CD} 34^{+} \mathrm{CD} 38^{-}$AML cells $(74.0 \pm 25.3 \%$ in 19 of 29 cases), whereas only a few cells $(4.9 \pm 1.6 \%)$ in the normal human stem cell (HSC)-enriched population $\left(\mathrm{Lin}^{-}\right.$ $\mathrm{CD} 34^{+} \mathrm{CD} 38^{-} \mathrm{CD} 90^{+}$) express weakly CD96. To examine whether $\mathrm{CD} 96^{+} \mathrm{AML}$ cells are enriched for LSC activity, AML cells were separated into $\mathrm{CD} 96^{+}$and $\mathrm{CD} 96^{-}$fractions and transplanted into irradiated newborn Rag2 ${ }^{-/-} \gamma_{c}^{-/-}$mice. In four of five samples, only $\mathrm{CD} 6^{+}$cells showed significant engraftment in bone marrow of the recipient mice. These results demonstrate that CD96 is a cell surface marker present on many AML-LSC and may serve as an LSC-specific therapeutic target. CD96 mini-antibodies, such as single chain variable fragments $(\mathrm{scFv})$ coupled to human Fc parts, are currently prepared in Gramatzki's laboratory for testing in clinical trials.
Dendritic cells (DC) have been extensively used in immunotherapeutic approaches upon genetic modification or loading with proteins, peptides or RNA, respectively. Among the different DC subsets that have been defined and are currently applied for immunotherapy, Marc Schmitz (Institute of Immunology, Technical University of Dresden, Dresden, Germany) presented the results obtained with the so-called slanDC. This subpopulation represents $0.5-2 \%$ of peripheral blood mononuclear cells and is characterised by the 6-sulfo LacNAc carbohydrate modification of the P-selectin glycoprotein ligand 1. Upon in vitro culture they undergo spontaneous maturation in the absence of exogeneous cytokines and secrete large amounts of TNF- $\alpha$ and IL-12 after LPS stimulation. Moreover, slanDC pulsed with peptides derived from the tumor-associated prostate stem cell antigen are able to generate tumor-reactive $\mathrm{CD} 8^{+} \mathrm{CTLs}$ from the blood of tumor patients. In addition, slanDCs efficiently induce neoantigen-specific $\mathrm{CD}^{+} \mathrm{T}$ cells and direct the differentiation of naïve $\mathrm{CD}^{+} \mathrm{T}$ cells into $\mathrm{Th} 1$ cells upon LPS stimulation.

Further studies reveal that slanDCs are able to exhibit direct antitumor effects. Thus, they mediate tumor-directed antibody-dependent cellular cytotoxicity, which is critically dependent on the engagement of the $\mathrm{Fc}-\gamma$ receptors $\mathrm{CD} 16$ and CD32. In addition, IFN- $\gamma$-activated slanDCs can efficiently lyse different tumor cell lines in the absence of antibodies. When focusing on the interaction between slanDCs and NK cells, recent results indicate that slanDCs profoundly enhance the IFN- $\gamma$ secretion and tumor-directed cytotoxicity of NK cells, which are critically dependent on IL-12. Reciprocally, NK cells efficiently promote DC maturation and strongly augment IL-12 secretion by slanDCs. IFN- $\gamma$ released by NK cells contribute to this effect. These results provide evidence that slanDCs may play a pivotal role in triggering innate and adaptive antitumor immunity and may represent attractive candidates for immunotherapy of tumors.

Another concept to enhance immune response was introduced by Hinrich Abken (University of Cologne, Germany) who shifted the attention to $\mathrm{T}$ cells for tumor immunotherapy and gave a summary on the use of chimeric $\mathrm{T}$ cell receptors (TCR) consisting of an intracellular domain of the signal transducing $\mathrm{CD} 3 \zeta$ chain fused to a single chain antibody fragment $(\mathrm{scFv})$ directed against different antigens. Modified T cells expressing these receptors attack their target cells, but the position of the binding epitope is important for the activation capacity. Interestingly, an increased binding affinity of the $\mathrm{scFv}$ does not necessarily affect the receptor-mediated activation.

To investigate the influence of costimulatory signals on chimeric TCR, the intracellular domain of $\mathrm{CD} 28$ was added. Using the $\mathrm{CD} 3 \zeta-\mathrm{CD} 28$ receptor he could show that the affinity-dependent threshold quality in T cell activation 
was not altered by the costimulatory domain, whereas the $\mathrm{T}$ cell activation was increased as determined by higher IFN- $\gamma$ and IL-2 levels and a decreased viability of target cells. Moreover, the $\mathrm{CD} 3 \zeta$-CD28 receptor signalling is resistant to the TGF- $\beta$-mediated suppression also resulting in killing of TGF- $\beta$ secreting tumor cells. For this effect the lck binding site within the CD28 domain was important as demonstrated by mutation experiments.

Effector $\mathrm{T}$ cells are controlled and repressed by Treg through an IL-2-dependent mechanism. Therefore, $\mathrm{T}$ cells were transfected with a recombinant immunoreceptor that drives CD28 costimulation with reduced IL-2 induction. Upon antigen-induced activation these cells produce less IL-2 and obtain a resistance against repression by Tregs, whereas the IFN- $\gamma$ secretion capacity to control tumor growth was affected. This improved knowledge will now be used in a multi-center phase I trial of $\mathrm{CD}^{+} 0^{+}$cutaneous $\mathrm{T}$ cell lymphoma. $\mathrm{T}$ cells from these patients will be modified by viral transfections of the chimeric TCR expanded and then reinfused in vivo to evaluate the influence on tumor growth.

An additional strategy of passive adoptive immunotherapy consists in the injection of mAbs. In his talk Matthias Peipp (Christian-Albrechts-University, Division of Stem Cell Transplantation and Immunotherapy, Kiel, Germany) gave an interesting overview of the evolution and optimisation of antibodies for such an aim. In the early phase of clinical implementation murine antibodies have been used, whereas in the last decade chimerized and finally fully human antibodies have been developed. These antibodies, mainly of the IgG subtype, are currently employed in various clinical trials.

However, for the development of effective antibodies their mechanism of activity had to be taken in to account, which could be mediated by cross linking and neutralizing soluble factors via Fab-associated direct effector mechanisms, Fc-mediated indirect effector mechanisms (ADCC) and pharmacokinetics. In ADCC the affinities of different IgG subclasses for their specific activating versus inhibiting $\mathrm{Fc}$ receptors are of enormous relevance, thus making their manipulation an important aim of antibody engineering. As an example Peipp showed some data using a Fc-engineered CD19 antibody, which represents an ideal target antigen on malignant $\mathrm{B}$ cells due to its long-lasting expression during B cell development. Interestingly, the non-engineered mouse CD19 antibody did not efficiently induce ADCC of lymphoma cells. In contrast, after humanisation and modification of two amino acids in the Fc part, the modified antibody (Xmab-CD19; Xencor Inc., Monrovia, CA, USA) exhibits an increased lysis of target cells, even in comparison to Rituximab, an antibody directed against CD20 that is currently used in non-Hodgkin lymphoma therapy. Furthermore different leukemic and lymphatic tumor cells are more efficiently lysed by the modified CD19 antibody via ADCC.

In addition to the protein-engineering, glyco-engineering comes into the focus of research. It is known that glycosylation of $\mathrm{IgG}-\mathrm{Fc}$ is essential for recognition and activation via Fc receptors. In his talk M. Peipp provided data about the functional comparison between the wt glycosylated form and a $30 \%$ reduced fucose residues form of an antibody targeting the epidermal growth factor receptor. The "low fucose" antibody exhibited a more effective mononuclear cell (MNC)-mediated killing compared to the wt antibody. In contrast the lytic efficacy of the "low fucose" variant diminished in polymorphonuclear (PMN) leukocyte-mediated killing revealing that different effector cells showed variable responses to antibody modifications [17]. In addition, a comparative analysis of the activity of protein- and glyco-engineered antibodies directed against CD20 demonstrated that both improvements resulted in an efficient ADCC enhancement of modified versus wt mAb, whereas double engineered (protein and glyco) antibodies exhibit no synergistic effects on ADCC induction. Thus, Peipp concluded that engineered antibodies can potentially enhance NK cell-mediated lysis, although controlled clinical trials with these antibodies still have to demonstrate their superior activity.

During the meeting many clinical trials or preclinical testing of peptide vaccination have been presented. Shifting to the clinical setting, different approaches have been proposed belonging to passive and active immunotherapy. An example of combinatorial therapy in which antigen immunization is associated with $\mathrm{T}_{\text {reg }}$ depletion was reported by Licia Rivoltini (Istituto Nazionale dei Tumori, Milan, Italy). She summarized data obtained in an ongoing twoarmed phase II trial of high risk melanoma patients. HLAA*0201 patients were subcutaneously immunised with four modified peptides derived from MelanA/Mart-1, Gp100, NY-ESO and Survivin, emulsified in Montanide, and administered in combination with low dose of cyclophosphamide and IL-2. The peripheral blood leukocytes of these patients were monitored over a period of 36 months for immune response by IFN- $\gamma$ ELISPOT analysis, HLA tetramer staining and $\mathrm{T}_{\text {reg }}$ analysis. At May 2008, 43 patients were enrolled, 22 in the control arm and 21 in the vaccination arm. Surprisingly, the frequency of $\mathrm{CD}^{+} \mathrm{CD} 25^{+}$ Foxp $3^{+} \mathrm{T}_{\text {reg }}$ cells, that in these early patients is comparable to that of healthy donors, does not chance during vaccine treatment, suggesting that the used schedule of cyclophosphamide ( $300 \mathrm{mg} / \mathrm{sqm} /$ every 2 weeks, for three times) is unable to affect the number of peripheral $\mathrm{T}_{\text {regs }}$. In contrast, the administration of IL-2 is more critical leading to a transient expansion of $\mathrm{CD}^{+} \mathrm{CD}^{+} 5^{+}$Foxp $^{+} \mathrm{T}_{\text {reg }}$ cells, that however secrete IFN- $\gamma$ and not $\mathrm{TGF} \beta$ and may thus represent effector rather than suppressive $\mathrm{T}$ cells. At opposite to $\mathrm{T}_{\text {reg }}$, 
MDSC $\left(\mathrm{CD} 14^{+}\right.$HLA-DRneg/low) were already increased in peripheral blood of early melanoma patients, and they were not affected by cyclophosphamide administration. However, the vaccine strategy employed increased the frequency and the activation state of peptide/melanoma-specific $\mathrm{T}$ cells in $60-90 \%$ of vaccinated patients (depending on the peptide) in peripheral blood and in vaccine-draining lymph nodes. It is noteworthy that with the exception of MART-1 modified peptides exhibit a reduced efficacy to induce $\mathrm{T}$ cells able to cross-react with the naturally processed peptide expressed by melanoma cells.

In line with the problem of inducing an immune response in the presence of an established tumor with all its possible escape mechanisms, Pier-Luigi Lollini (University of Bologna, Italy) focussed his talk on tumor immunoprevention and therapy against metastasis development, both settings without extensive immunosuppressive conditions. As a system the BalbNeuT mouse model was chosen in which the rat HER-2/neu oncogene is expressed under the Mouse Mammary Tumor Virus (MMTV) promoter leading to the development of carcinomas in all ten mammary glands within 5 months of life.

Chronic vaccination of female BalbNeuT mice with a "triplex" vaccine, consisting of an allogeneic NeuT transgenic cell line derived secreting IL12p70, results in complete prevention of tumor development mainly through antibody responses against HER-2/neu that mediated complement-induced or cell-mediated cytotoxicity against tumor cells, but can also act as receptor antagonists inhibiting signal transduction and inducing receptor recycling. Moreover, IFN- $\gamma$ induced by the vaccine-secreted IL-12 inhibits mammary gland growth and impairs neoangiogenesis necessary for tumor development. The triplex vaccine demonstrated its efficacy not only in the cell-based form, but also as a DNA vaccine consisting of plasmids encoding the allogeneic H-2-Dq molecule, the HER-2/neu antigen and the IL12p70 heterodimer. Despite similar protection, different levels of IgG subclasses were found in the serum and only IFN- $\gamma$, but not IL-4 is produced upon in vitro restimulation using the DNA-based vaccine. The potency of the triplex vaccine was also retained in an immunotherapy protocol against metastasis. Combination of the triplex vaccine with the depletion of $\mathrm{T}_{\text {reg }}$ further improved the therapy efficacy, thus confirming the possibility to induce an immune response in tumor bearing host and correlating its efficacy with immunosuppressive conditions [15].

In order to mimic a more clinical-like situation, the triplex vaccine was administrated to BALBp53Neu mice that in addition to the HER-2/neu oncogene carry one copy of the p53 oncosuppressor gene. Whereas in control mice the tumor development was associated with the loss of the second copy of the p53 gene, vaccination with the triplex vaccine prevented the oncogene-driven tumor expansion thereby limiting the accumulation of other mutations, like p53 loss.

The success of such a vaccine, might be due to the "oncoantigen" phenotype of the HER-2/neu molecule, i.e. its antigenic nature together with the "addiction" of the tumor to its expression for development. However, it is noteworthy to underline that in the group of Guido Forni, the development of mammary tumor in BalbNeuT mice was prevented by a vaccine targeting not the HER-2/neu antigen.

Searching for tumor antigens with low possibility of immune selection, Per thor Straten (Center for Cancer Immune Therapy (CCIT), University Hospital, Herlev, Denmark) employed proteins involved in apoptosis regulation (survivin, Bcl-2, Bcl-XL), in drug resistance (TRAG-3) as well as in metastasis (RhoC). Survivin belongs to the inhibitors of apoptosis (IAP) gene family and is abundantly and ubiquitously present during development, whereas in normal adult tissues it is undetectable, but prominently re-expressed in virtually every human cancer. Molecular profiling demonstrated that survivin overexpression is consistently associated with advanced disease, high grade, reduced patient survival, resistance to therapy, and accelerated recurrences, thus defining survivin as a prognostic and predictive marker. Moreover, survivin may serve as potential target for immune-mediated tumor destruction in cancer patients since its down-regulation would severely inflict the survival capacity of tumor cells and abrogate immune selection. In addition to survivin other regulators of apoptosis, such as Bcl-2 or Bcl-XL, were scanned by thor Straten's group for potential HLA-binding peptides, and consecutively analysed in $\mathrm{T}$ cell assays (ELISPOT, tetramer) for spontaneous reactivity in patients' blood samples. The ex vivo lytic capacity of peptide-specific T cells as well as their TCR repertoire was explored in cytotoxicity assays or by TCR clonotype mapping, respectively and compared to that of established antigens in melanoma, such as MART-1 and gp100. CTL with a high affinity to the MART-1 peptide paired with the lowest capacity to kill melanoma cells. This discrepancy could be explained by the low abundancy of MART-1 peptides on the surface of melanoma cell lines, and represents only one example of the difficulties in monitoring an immunological anti-tumor response.

Since induced biological responses are of quite low frequency, thor Straten suggested a combination of several different methods for monitoring immune reactivity against tumor cells. With ML-IAP (melanoma inhibitor of apoptosis protein) as candidate tumor antigen, a CTL response was not only detected in cancer patients, but also in approximately $10 \%$ of healthy donors. Search for sequence homology revealed similarities of ML-IAP with SS-56, a known cellular target of autoantibody response in patients with systemic Lupus erythematosus and Sjoegren's syndrome 
causing a cross-reactivity of T cells specific for SS-56 with ML-IAP thereby pointing to the risk of autoimmunity induction after vaccination of patients with tumor antigens. Otherwise, tolerance mechanisms could prevent a strong anti-tumor response after vaccination for tumor antigens as "self" proteins. In the last part of his talk, P-T. Straten addressed the suppressive molecule indoleamine 2,3-dioxygenase (IDO) as immunological target. IDO is not only expressed by APC creating a tolerogenic microenvironment, but also in the tumor itself. Indeed, IDO-derived peptides are recognized by host $\mathrm{CD} 8^{+} \mathrm{T}$ cells, which are able to kill tumor cell targets as well as mature DC. Thus, approaches targeting IDO-expressing cells by immunotherapy could lead to synergistic effects by killing not only tumor cells, but also cells responsible for induction of immune suppression.

Gustav Gaudernack (Rikshospitalet Montebello, Oslo, Norway) employed telomerase reverse transcriptase (hTERT) as target antigen due to its universal expression in human cancer cells and its critical functional role in tumor growth and development. GV1001 (hTERT611-626) is a promiscuous HLA class II epitope that can thus be used as a general cancer vaccine without needing HLA typing of the patient. A phase I/II study with 38 patients with non-resectable pancreatic cancer was conducted to investigate the safety, tolerability, and immunogenicity of intradermal vaccination with the telomerase peptide GV1001 at three dose levels in combination with granulocyte-macrophage colony-stimulating factor (GM-CSF). Monthly booster vaccinations were given as follow-up treatment. Beside the well toleration of GV1001, immune responses, detected as delayed-type hypersensitivity skin reaction and in vitro $\mathrm{T}$ cell proliferation, were observed in 24 of 38 patients.

In line with the idea that for effective immunotherapy an "oncoantigen" should be used as target, Carmen Scheibenbogen (Charité, Berlin, Germany) focused her attention on the transcription factor PAX2 that is normally expressed in the embryo during kidney and eye development, but is also expressed in tumors of different histotypes and is involved in the regulation of the Wilm's tumor (WT1) antigen, a known tumor-associated antigen with a good correlation between specificity of expression and essentiality in function. Using different prediction algorithms to take into account HLA-binding motifs and proteasome cleavage preferences, possible PAX2 epitopes were synthesised and used to stimulate peripheral blood mononuclear cells (PBMC) from healthy volunteers and tumor-bearing patients. Only PBMC from the latter responded to PAX2 peptides with production of IFN- $\gamma$ or TNF- $\alpha$, thus indicating the tumor-restricted expression of the antigen and its applicability for tumor therapy. By cytotoxic experiments of cold target inhibition using $\mathrm{T} 2$ cells pulsed with the different peptides, one of the predicted eptiope, namely
PAX2 337-345, appeared to be not only a CTL epitope, but also a natural HLA ligand.

Exactly on the concept of natural HLA ligands the strategy presented by Stefan Stevanovic (University of Tübingen, Institute of Immunology, Tübingen, Germany) is based. In contrast to the so-called CTL epitopes that are identified by their ability to induce cell lysis upon pulsing of professional antigen presenting cells the natural ligands are peptides processed by the tumor cells and presented on the cell surface. In the therapeutic approach biopsies from tumors and normal tissues of renal cell carcinoma (RCC) patients are "stripped" of their natural HLA ligands, that are then analysed by mass spectrometry for identification and evaluated for possible usage in a peptide-based vaccine upon comparison of expression among normal and tumor tissue. Preliminary results in one RCC patient vaccinated with a cocktail of 3 HLA class I ligands together with 3 HLA class II ligand-epitopes identified in this way provide promising results since the patient is still tumor free. Initial comparison among the natural HLA ligands obtained from different RCC patients revealed only a few common peptides, thus suggesting a high level of individuality, even if the limitation in the techniques that allows the identification of $10 \%$ of the total stripped peptides can also be one reason for this low level of overlap.

Taken together, the intersectional work between the avenues of stem cell biology, oncology and tumor immunology promises not only a detour-free access to an understanding of the biology of tumors, but will also pave yet narrow paths for clinical applications.

\section{References}

1. Aboody KS, Najbauer J, Danks MK (2008) Stem and progenitor cell-mediated tumor selective gene therapy. Gene Ther 15:739752

2. Aptsiauri N, Cabrera T, Garcia-Lora A, Lopez-Nevot MA, RuizCabello F, Garrido F (2007) MHC class I antigens and immune surveillance in transformed cells. Int Rev Cytol 256:139-189

3. Boman BM, Wicha MS (2008) Cancer stem cells: a step toward the cure. J Clin Oncol 26:2795-2799

4. Brune M, Castaigne S, Catalano J, Gehlsen K, Ho AD, Hofmann WK, Hogge DE, Nilsson B, Or R, Romero AI, Rowe JM, Simonsson B, Spearing R, Stadtmauer EA, Szer J, Wallhult E, Hellstrand K (2006) Improved leukemia-free survival after postconsolidation immunotherapy with histamine dihydrochloride and interleukin-2 in acute myeloid leukemia: results of a randomized phase 3 trial. Blood 108:88-96

5. Dong X, An B, Salvucci Kierstead L, Storkus WJ, Amoscato AA, Salter RD (2000) Modification of the amino terminus of a class II epitope confers resistance to degradation by CD13 on dendritic cells and enhances presentation to T cells. J Immunol 164:129 135

6. Gamzatova Z, Villabona L, van der Zanden H, Haasnoot GW, Andersson E, Kiessling R, Seliger B, Kanter L, Dalianis T, Bergfeldt K, Masucci GV (2007) Analysis of HLA class I-II 
haplotype frequency and segregation in a cohort of patients with advanced stage ovarian cancer. Tissue Antigens 70:205-213

7. Hanson MG, Ozenci V, Carlsten MC, Glimelius BL, Frodin JE, Masucci G, Malmberg KJ, Kiessling RV (2007) A short-term dietary supplementation with high doses of vitamin $\mathrm{E}$ increases NK cell cytolytic activity in advanced colorectal cancer patients. Cancer Immunol Immunother 56:973-984

8. Kucia M, Halasa M, Wysoczynski M, Baskiewicz-Masiuk M, Moldenhawer S, Zuba-Surma E, Czajka R, Wojakowski W, Machalinski B, Ratajczak MZ (2007) Morphological and molecular characterization of novel population of CXCR4+ SSEA-4+ Oct$4+$ very small embryonic-like cells purified from human cord blood: preliminary report. Leukemia 21:297-303

9. Luo W, Ko E, Hsu JC, Wang X, Ferrone S (2006) Targeting melanoma cells with human high molecular weight-melanoma associated antigen-specific antibodies elicited by a peptide mimotope: functional effects. J Immunol 176:6046-6054

10. Malmberg KJ, Arulampalam V, Ichihara F, Petersson M, Seki K, Andersson T, Lenkei R, Masucci G, Pettersson S, Kiessling R (2001) Inhibition of activated/memory $(\mathrm{CD} 45 \mathrm{RO}(+)) \mathrm{T}$ cells by oxidative stress associated with block of NF-kappaB activation. J Immunol 167:2595-2601

11. Malmberg KJ, Lenkei R, Petersson M, Ohlum T, Ichihara F, Glimelius B, Frodin JE, Masucci G, Kiessling R (2002) A shortterm dietary supplementation of high doses of vitamin $\mathrm{E}$ increases $\mathrm{T}$ helper 1 cytokine production in patients with advanced colorectal cancer. Clin Cancer Res 8:1772-1778

12. Mueller LP, Luetzkendorf J, Mueller T, Reichelt K, Simon H, Schmoll HJ (2006) Presence of mesenchymal stem cells in human bone marrow after exposure to chemotherapy: evidence of resistance to apoptosis induction. Stem Cells 24:2753-2765

13. Mueller T, Mueller LP, Luetzkendorf J, Voigt W, Simon H, Schmoll HJ (2006) Loss of Oct-3/4 expression in embryonal carcinoma cells is associated with induction of cisplatin resistance. Tumor Biol 27:71-83

14. Nakamizo A, Marini F, Amano T, Khan A, Studeny M, Gumin J, Chen J, Hentschel S, Vecil G, Dembinski J, Andreeff M, Lang FF (2005) Human bone marrow-derived mesenchymal stem cells in the treatment of gliomas. Cancer Res 65:3307-3318

15. Nanni P, Nicoletti G, Palladini A, Croci S, Murgo A, Antognoli A, Landuzzi L, Fabbi M, Ferrini S, Musiani P, Lezzi M, De Giovanni C, Lollini PL (2007) Antimetastatic activity of a preventive cancer vaccine. Cancer Res 67:11037-11044

16. Norell $\mathrm{H}$, Carlsten $M$, Ohlum $T$, Malmberg KJ, Masucci G, Schedvins K, Altermann W, Handke D, Atkins D, Seliger B, Kies- sling R (2006) Frequent loss of HLA-A2 expression in metastasizing ovarian carcinomas associated with genomic haplotype loss and HLA-A2-restricted HER-2/neu-specific immunity. Cancer Res 66:6387-6394

17. Peipp M, Lammerts van Bueren JJ, Schneider-Merck T, Bleeker WW, Dechant M, Beyer T, Repp R, van Berkel PH, Vink T, van de Winkel JG, Parren PW, Valerius T (2008) Antibody fucosylation differentially impacts cytotoxicity mediated by NK and PMN effector cells. Blood 19

18. Ratajczak MZ, Zuba-Surma EK, Machalinski B, Ratajczak J, Kucia M (2008) Very small embryonic-like (VSEL) stem cells: purification from adult organs, characterization, and biological significance. Stem Cell Rev 4(2):89-99

19. Ratajczak MZ, Zuba-Surma EK, Wysoczynski M, Ratajczak J, Kucia M (2008) Very small embryonic-like stem cells: characterization, developmental origin, and biological significance. Exp Hematol 36:742-751

20. Sackstein R, Merzaban JS, Cain DW, Dagia NM, Spencer JA, Lin CP, Wohlgemuth R (2008) Ex vivo glycan engineering of CD44 programs human multipotent mesenchymal stromal cell trafficking to bone. Nat Med 14:181-187

21. Studeny M, Marini FC, Champlin RE, Zompetta C, Fidler IJ, Andreeff M (2002) Bone marrow-derived mesenchymal stem cells as vehicles for interferon-beta delivery into tumors. Cancer Res 62:3603-3608

22. Takahashi A, Hanson MG, Norell HR, Havelka AM, Kono K, Malmberg KJ, Kiessling RV (2005) Preferential cell death of CD8 + effector memory (CCR7-CD45RA-) T cells by hydrogen peroxide-induced oxidative stress. J Immunol 174:6080-6087

23. Wang E, Worschech A, Marincola FM (2008) The immunologic constant of rejection. Trends Immunol 29:256-262

24. Wang X, Ko EC, Peng L, Gillies SC, Ferrone S (2005) Human high molecular weight melanoma-associated antigen mimicry by mouse anti-idiotypic monoclonal antibody MK2-23:enhancement of immunogenicity of anti-idiotypic monoclonal antibody MK223 by fusion with interleukin 2. Cancer Res 65:6976-6983

25. Wulfaenger J, Niedling S, Riemann D, Seliger B (2008) Aminopeptidase N (APN)/CD13-dependent CXCR4 downregulation is associated with diminished cell migration, proliferation and invasion. Mol Membr Biol 25:72-82

26. Wang Z, Zhang L, Qiao A, Watson K, Zhang J, Fan GH (2008) Activation of CXCR4 triggers ubiquitination and down-regulation of major histocompatibility complex class I (MHC-I) on epithelioid carcinoma HeLa cells. J Biol Chem 15(283):3951-3959 